\title{
Excitonic quasimolecules in nanosystems containing quantum dots
}

Sergey I. Pokutnyi ${ }^{*}$, Wlodzimierz Salejda*

${ }^{*}$ Chuiko Institute of Surface Chemistry, National Academy of Sciences of Ukraine,

17 General Naumov Str., UA - 03164 Kyiv, Ukraine

e-mail: Pokutnyi_Sergey@inbox.ru; pokytniy@mail.ua

** Wroclaw University of Technology,

27 Wyb. Wyspianskiego,

50370 Wroclaw, Poland

e-mail: wlodzimierz.salejda@pwr.edu.pl

\begin{abstract}
The possibility of occurrence of the excitonic quasimolecule formed of spatially separated electrons and holes in a nanosystem that consists of $\mathrm{CuO}$ quantum dots synthesized in a silicate glass matrix. It is shown that the major contribution to the excitonic quasimolecule binding energy is made by the energy of the exchange interaction of electrons with holes and this contribution is much more substantial than the contribution of the energy of Coulomb interaction between the electrons and holes.
\end{abstract}

Keywords: Spatially separated electron and holes, Quantum dots, Binding energy, Quasiatomic nanosystems.

\section{Council for Innovative Research}

Peer Review Research Publishing System

\section{Journal: Journal of Advances in Chemistry}

Vol. 12, No. 2

editor@cirjac.com

www.cirjac.com 


\section{Introduction}

The optical properties of quasi-zero-dimensional semiconductor nanosystems that consist of spherically shaped semiconductor quantum dots (QDs) grown to a radius of $a \approx(1-30) \mathrm{nm}$ in transparent insulator matrices are defined in a large extent by the energy spectra of charge carriers localized close to the QD surface [1-5]. In Refs [1, 2], the optical properties of silicate glassy samples containing CuO QDs were experimentally studied. The average radii of such QDs were in the range of $a \approx(2-30) \mathrm{nm}$.

In Refs [3-5], it was found that an electron could be localized in a polarization well near the external QD surface, while a hole was in motion within the QD. In previous papers, it was theoretically established [6, 7] that the shift of the lowtemperature absorption spectral peak in such a nanosystem [3] was due to quantum confinement of the energy of the exciton ground state (formed from a spatially separated hole and electron).

In Refs [1-5], a shift of the spectral peak of the low-temperature absorption was observed also for samples with a QD content of $x \approx(1-2) \%$. It was noted that, at such a QD content in the samples, one must take into account the interaction between charge carriers localized above the QD surfaces [1 - 5].

Therefore, in this study, we develop the theory of a excitonic quasimolecules (formed from spatially separated electrons and holes) in a nanosystem that consists of CuO QDs synthesized in a silicate glass matrix. By using the variational method, we obtain the total energy and the binding energy of the biexciton singlet ground state in such system as a function of the spacing between the QD surfaces and of the QD radius. Moreover we establish that the spectral shift of the low-temperature absorption peak [1, 2] in such a nanosystem is due to quantum confinement of the energy of the biexciton ground state.

\section{Excitonic Quasimolecules Formed From Spatially Separated Electrons and Holes}

We model our nanosystem as consisting of two spherical semiconductor QDs, $A$ and $B$, synthesized in a borosilicate glass matrix with the permittivity $\varepsilon_{1}$. Let the QD radii be $a$, the spacing between the spherical QD surfaces be $D$. Each QD is formed from a semiconductor material with the permittivity $\varepsilon_{2}$. For simplicity and without loss of generality, we assume that the holes $h(A)$ and $\quad h(B)$, with the effective masses $m_{h}$, are in the QD (A) and QD (B) centers and the electrons $e(1)$ and $e(2)$, with the effective masses $m_{e}^{(1)}$, are localized near the spherical $Q D(A)$ and $Q D(B)$ surfaces, respectively. The above assumption is reasonable, since the ratio between the effective masses of the electron and hole in the nanosystem is much smaller than unity: $\left(\left(m_{e}^{(1)} / m_{h}\right) \ll 1\right)$. Let us assume that there is an infinitely high potential barrier at the spherical QD - matrix interface. Therefore, in the nanosystem, holes do not leave the QD bulk, whereas electrons do not penetrate into the QDs.

The characteristic lengths of the problem are determined by the quantities

$$
a_{h}=\varepsilon_{2} \hbar^{2} / m_{h} e^{2}, a_{e}^{(1)}=\varepsilon_{1} \hbar^{2} / m_{e}^{(1)} e^{2}, a_{e x}^{0}=\frac{2 \varepsilon_{1} \varepsilon_{2}}{\left(\varepsilon_{1}+\varepsilon_{2}\right)} \cdot \frac{\hbar^{2}}{\mu_{0} e^{2}}
$$

where $a_{h}$ and $a_{e}^{(1)}$ are the Bohr radii of holes and electrons, in the semiconductor with the permittivity $\varepsilon_{2}$ and in the matrix with the permittivity $\varepsilon_{1}$, respectively; $a_{e x}^{0}$ is the Bohr radius of a two-dimensional (2D) exciton localized above the planar interface between a semiconductor with the permittivity $\varepsilon_{2}$ and the matrix with the permittivity $\varepsilon_{1}$ (the hole is in motion within the semiconductor, whereas the electron is in the matrix), (e) is the electron charge, $\mu_{0}=m_{e}^{(1)} m_{h} /\left(m_{e}^{(1)}+m_{h}\right)$ is the reduced $2 \mathrm{D}$ - exciton effective mass. The fact that all the characteristic lengths of the problem

$$
a, D, a_{e}^{(1)}, a_{h}, a_{e x}^{0} \gg a_{0}
$$

are much larger than the inter-atomic spacing $a_{0}$ allows us to consider the motion of electrons and holes in the nanosystem in the effective mass approximation [6,7].

In the context of both the adiabatic approximation and the effective mass approximation, by using the variational method in analogy with Ref. [8], we get the total energy $E_{0}(\widetilde{D}, \tilde{a})$ and the binding energy $E_{b}(\widetilde{D}, \tilde{a})$ of the biexciton singlet ground state (in which the spins of the electrons $e(1)$ and $e(2)$ are antiparallel) as a function of the spacing between the QD surfaces $D$ and the QD radius a:

$$
E_{0}(\tilde{D}, \tilde{a})=2 E_{e x}(\tilde{a})+E_{\text {s }}(\tilde{D}, \tilde{a})
$$

where $\widetilde{D}=\left(D / a_{e x}^{0}\right)$ and $\tilde{a}=\left(a / a_{e x}^{0}\right)$. In Eq. (3), the binding energy $E_{e x}(\tilde{a})$ of the ground state of the exciton (formed from an electron and a hole spatially separated from the electron) localized above the $\mathrm{QD}(\mathrm{A})$ (or $\mathrm{QD}(\mathrm{B})$ ) surface is determined as shown in Ref.s [6,7]. For the nanosystem under study, the values of the binding energies $E_{\text {ex }}(\tilde{a})$ have been calculated in Ref.s [6,7] for the experimental conditions of Ref.s [1, 2]. In these experimental works the silicate glassy samples doped with $\mathrm{CuO}$ up to a content $x=2 \%$, were produced by the sol-gel technique [1, 2]. 
In [1, 2], the optical properties of silicate glassy samples containing CuO QDs were studied experimentally. The average radii of such QDs were in the range of

$$
2 \leq a \leq 30(\mathrm{~nm})
$$

The results of the variational calculation of the binding energy $E_{b}(\tilde{D}, \tilde{a})$ of the biexciton singlet ground state in the nanosystem of CuO QDs synthesized in a silicate glass matrix, by choosing an average radii of $\bar{a}_{1}=4.4 \mathrm{~nm}$ are shown in Fig.1. The value of $\bar{a}_{1}=4.4 \mathrm{~nm}$ is in the range of the average CuO QDs radii a studied in the experimental conditions $[1,2]$. At a QD content of ( $x$ or $0.6 \%$ ), one must take into account the interaction of charge carriers localized above the QD surfaces.

The binding energy $E_{b}(\tilde{D}, \tilde{a})$ of the biexciton singlet ground state in the nanosystem containing CuO QDs with average radii of $\bar{a}_{1}=4.4 \mathrm{~nm}$ shows a minimum of $E_{b}^{(1)}\left(D_{1}, \bar{a}_{1}\right) \approx-91.44 \mathrm{me} V$ (at the spacing $D_{1}=3.0 \mathrm{~nm}$ ) (see Fig. 1) This value of $E_{b}^{(1)}$ corresponds to the temperature $T_{c}=1000 \mathrm{~K}$. From Fig.1, it follows that a biexciton (excitonic quasimolecule) is formed in the nanosystem, starting from a spacing between the QD surfaces $D \geq D_{c}^{(1)} \cong 2.2 n m$. The formation of such a excitonic quasimolecule is of the threshold character and possible only in a nanosystem with QDs with average radii $\bar{a}_{1}$ such that the spacing between the QD surfaces $D$ exceeds a certain critical spacing $D_{c}^{(1)}$. The emergence of critical spacing $D_{c}^{(1)}$ due to the fact that with decreasing distance $D$ quantum size effect leads to the destruction of the binding energy in the quasimolecule. Moreover, the exciton quasimolecule can exist only at temperatures below a certain critical temperature: $T_{c}=1000 \mathrm{~K}$.

As follows from the results of the variational calculation, the binding energy of an exciton (formed from an electron and a hole spatially separated from the electron) localized above the surface of the $\mathrm{QD}(\mathrm{A})$ (or a $\mathrm{QD}(\mathrm{B})$ ) with an average radius of $\bar{a}_{1}=4.4 \mathrm{~nm}$ is $\operatorname{Eex}\left(\bar{a}_{1}\right)=-1.102 \mathrm{eV}$. In this case the energy of the biexciton singlet ground state $E_{0}(\widetilde{D}, \tilde{a})(3)$ takes the value $E_{0}(\widetilde{D}, \tilde{a})=-2,295 \mathrm{eV}$. The value of $\bar{a}_{1}=4.4 \mathrm{~nm}$ is in the range of the average CuO QDs radii a (4) studied in the experimental conditions of $[1,2]$.

The variational method used here for calculating the binding energy $E_{e}(\widetilde{D}, \tilde{a}) \quad(3)$ of the biexciton ground state in the nanosystem is appropriate, if the biexciton binding energy $E_{e}(\widetilde{D}, \tilde{a}) \quad$ is small compared to the binding energy $E_{e x}(\tilde{a})$ of the exciton ground state $[6,7]$. In other words, the inequality

$$
\left(E_{\theta}(\widetilde{D}, \tilde{a}) / E_{e x}(\tilde{a})\right) \ll 1 .
$$

must be satisfied. It should be emphasized that the criterion (5) of the applicability of the variational method used here for calculating the biexciton binding energy $E_{e}(\widetilde{D}, \tilde{a})(3)$ is satisfied (the corresponding ratio (4) of the order is 0.08 ).

From the results of variational calculation of the exciton quasimolecule binding energy $E_{e}(\tilde{D}, \tilde{a})$, it follows that the major contribution to the binding energy (3) is made by the average energy of the exchange interaction of the electrons $e(1)$ and $e(2)$ with the holes $h(A)$ and $h(B)$. At the same time, the energy of Coulomb interaction makes a much smaller contribution of the biexciton binding energy $E_{e}(\tilde{D}, \tilde{a})(3)$.

The major contribution to the exchange - interaction energy is made by the energy of the exchange interaction of the electron $e(1)$ with the holes $h(B)$, as well as of the electron $e(2)$ with the holes $h(B)$, as well as of the electron $e(2)$ with the holes $h(A)$. The major contribution to the Coulomb - interaction energy is made by the energy of Coulomb interaction of the electron $e(1)$ with the holes $h(B)$, as well as of the electron $e(2)$ with the holes $h(A)$.

As the spacing $D$ between the $\mathrm{QD}(\mathrm{A})$ and $\mathrm{QD}(\mathrm{B})$ surfaces is increased, starting from $D \geq D_{c}^{(2)} \cong 11.4 n m$, the average Coulomb - interaction energy substantially desreases. In addition, because of the decrease in the overlapping of the electron wave function, the average exchange interaction energy substantially decreases as well. As a consequence, the average Coulomb - interaction energy and the average energy of the exchange interaction of the electrons $e(1)$ and $e(2)$ with the holes $h(A)$ and $h(B)$ sharply desrease in comparison with the exciton binding energy $E_{e x}(\tilde{a})[6,7]$, resulting in the decomposition of the biexciton in the nanosystem into two excitons (formed of spatially separated electrons and holes) localized above the $\mathrm{QD}(\mathrm{A})$ and $\mathrm{QD}(\mathrm{B})$ surfaces (see Fig.1).

It should be noted that the calculations of the binding energy $\left|E_{e}(\widetilde{D}, \tilde{a})\right|$ [Eq.(3)] of the biexciton ground state in the nanosystem are variational, and therefore, can apparently yield understated estimates of the biexciton binding 
energy $\left|E_{e}(\widetilde{D}, \tilde{a})\right|$. It seems likely that the calculated maximum value of the biexciton binding energy $\left|E_{e}^{(1)}\right|$ is underestimated as well.

\section{Correlation between the theory and the experiments}

In Ref.s [6, 7] a low-temperature absorption peak at $\bar{E} \approx 4.27 \mathrm{eV}$ (at a temperature of $T=4.5 \mathrm{~K}$ ) was observed in samples with a CuO QD content of $x \approx 0.6 \%$; this peak was below the band gap $\left(E_{g}=6.53 \mathrm{eV}\right)$ of the matrix $\mathrm{SiO}_{2}$. The shift of the $\bar{E}$ low-temperature absorption peak with respect to the band gap of the matrix $\mathrm{SiO}_{2}$ was $\Delta \bar{E}=\bar{E}-E_{g} \cong-2.26 \mathrm{eV}$.

By comparing the energy $E_{0}(\widetilde{D}, \tilde{a})$ (3) of the biexciton ground state with the shift $\Delta \bar{E} \cong-2.26 \mathrm{eV}$ of the absorption spectral peak, we obtain the average spacing $\bar{D}_{0} \approx 4.8 \mathrm{~nm}$ between the QD (A) and QD (B) surfaces (at an average QD radius of $\left.\bar{a}_{1}=4.4 \mathrm{~nm}\right)$. In this case, the biexciton binding energy is $E_{s}^{(2)}\left(\bar{D}_{0}, \bar{a}_{1}\right) \cong 54 \mathrm{meV}$, corresponding to a temperature of $T_{2} \approx 626 \mathrm{~K}$.

Therefore, the experimentally observed shift shift $\Delta \bar{E}$ of the low-temperature absorption peak in the samples containing CuO QDs with a content of $x \approx 0.6 \%[1,2]$ is due to the dependence of the energy $\left|E_{e}(\widetilde{D}, \tilde{a})\right|(3)$ of the singlet ground state of a biexciton (formed from spatially separated electrons and holes) on the spacing $D$ between the QD surfaces and on the $\mathrm{QD}$ radius $a$.

It should be noted that the calculations of the binding energy $\left|E_{e}(\widetilde{D}, \tilde{a})\right|(3)$ of the biexciton ground state in the nanosystem are variational, and therefore, can apparently yield understated estimates of the biexciton binding $\left|E_{e}(\widetilde{D}, \tilde{a})\right|$ (3). It seems likely that the calculated maximum value of the biexciton binding energy $\left|E_{e}^{(1)}\right|$ is underestimated as well.

\section{Conclusions}

The possibility of occurrence of the excitonic quasimolecule formed of spatially separated electrons and holes in a nanosystem that consists of $\mathrm{CuO}$ quantum dots synthesized in a silicate glass matrix. It is shown that the major contribution to the excitonic quasimolecules binding energy is made by the energy of the exchange interaction of electrons with holes and this contribution is much more substantial than the contribution of the energy of Coulomb interaction between electrons and holes.

It is established that, in a nanosystem composed of CuO QDs of an average radii of $\bar{a}_{1}$, the formation of a biexciton has a threshold character and it is possible only in a nanosystem, in which the spacing $D$ between the QD surfaces is larger than a certain critical spacing $D_{c}^{(1)}$. It is shown that an increase in the spacing $D$ between the QD surfaces from $D \geq$ $D_{c}^{(2)}$ yields decomposition of the biexciton into two excitons (formed from spatially separated electrons and holes) localized above the QD surfaces. Thus, a biexciton can be formed in the nanosystem under the condition $D_{c}^{(1)} \leq D \leq D_{c}^{(2)}$. In addition, the biexciton can exist only at temperatures below a certain critical temperature $T \mathrm{c}=1000 \mathrm{~K}$. In the $\mathrm{CuO}$ single crystal, the biexciton is formed with the binding energy $E_{b}=26 \mathrm{meV}$ corresponding to the temperature $292 \mathrm{~K}$. At the same time, the biexciton binding energy $E_{\beta}^{(1)}$ in the nanosystem is about an order of magnitude higher than $E_{b}$.

The binding energy of an exciton quasi molecule consisting of two CuO QDs, takes enormous value greater than the binding energy of the biexciton in $\mathrm{CuO}$ by almost two orders of magnitude. This effect makes it possible to use a quasimolecule exciton as an active medium nanolasers radiating in the infrared range of the spectrum, and working on the exciton transitions at room temperature, the quantum nano-element base, etc.

These results demonstrate the fundamental possibility of creating a new quasi-atomic nano - exciton quasi-molecules, including analogs of natural, with new physical properties. On this basis, possible construction of new quantum nano quasicrystals, management symmetry and lattice period which will allow to realize the unique physical and chemical effects and phenomena, to create new principles in material science.

\section{References}

[1] N.V. Borisenko, Modification of fumed silica copper acetylacetonate, Teor. Exper. Chemistry. 44 (2008) 191 - 195.

[2] N.V. Borisenko, Thermal conversion of copper acetylacetonate on the surface of fumed silica, Chemistry, Physics and Technology of the Surface. 4 (2013) $320-324$.

[3] V. Grabovskis, Y. Dzenis, A. Ekimov, Nonlinear- optical of semiconductor-doped glasses, Solid State Phys. 31 (1989) $272-274$.

[4] V.P. Dzyuba, A.E. Krasnok, J.N. Kulchin, Nonlinear- optical properties of the dielectric nanoparticles inserted into a dielectric matrix, Technical Physics Letters. 36 (2010) 1 - 4.

[5] J.N. Kulchin, V.P. Dzyuba, Optical properties of the dielectric nanoparticles inserted into a dielectric matrix, Pacific Science Rev. 12 (2010) 102 - 104.

[6] S.I. Pokutnyi, Binding energy of the exciton of a spatially separated electron and hole in quasi-zero-dimensional nanosystems, Technical Physics Letters. 39 (2013) $233-235$. 
[7] S.I. Pokutnyi, On an exciton with a spatially separated electron and hole in quasi-zero-dimensional nanostructures, Semiconductors. 47 (2013) 791 - 798.

[8] S.I. Pokutnyi, Biexciton formed from spatially separated electrons and holes in quasi-zero-dimensional nanosystems. Semiconductors. 47 (2013) $1626-1635$.

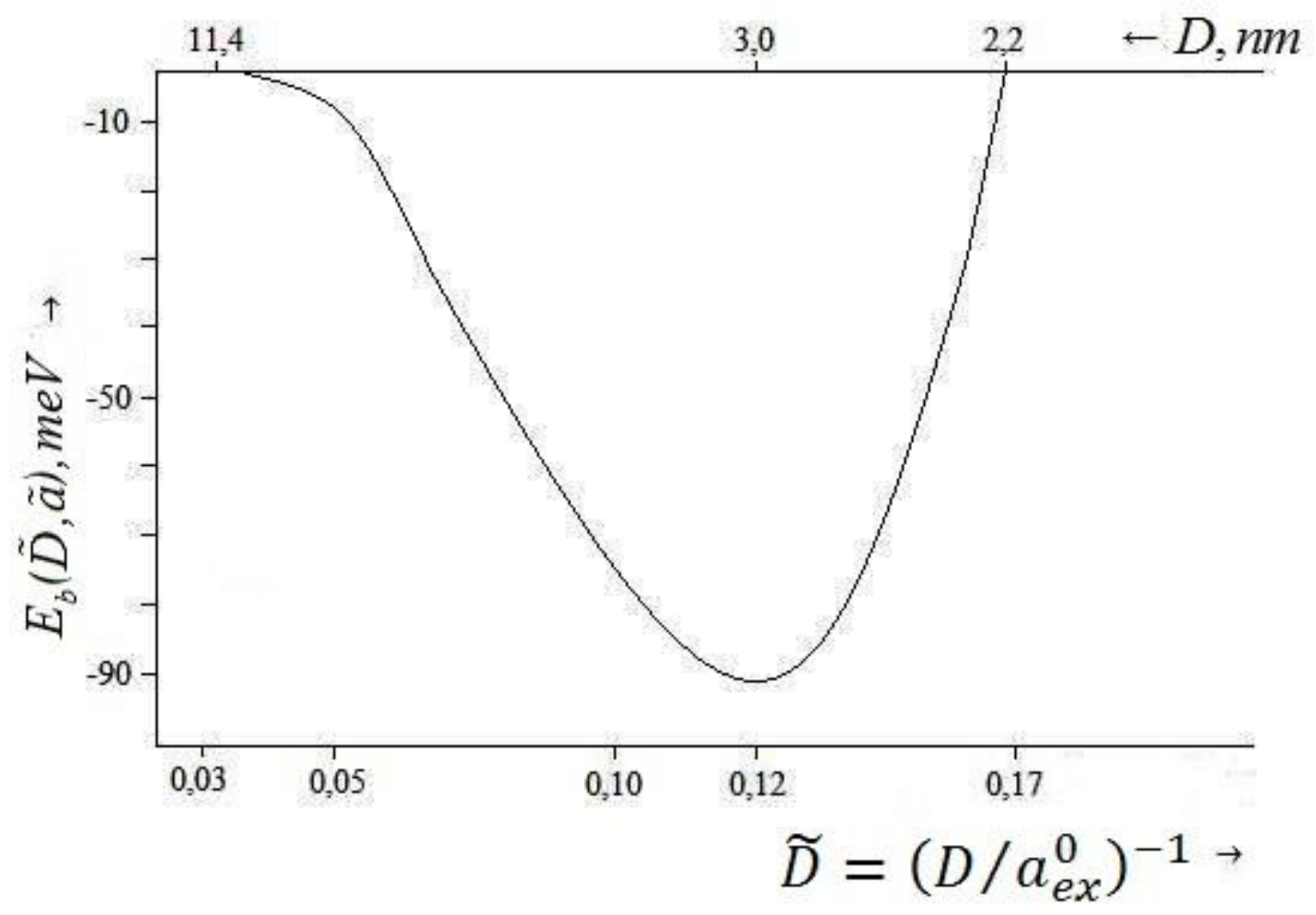

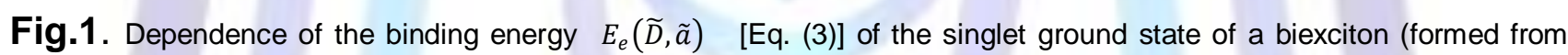
spatially separated electrons and holes) in the nanosystem consisting of two spherical CuO QDs, QD (A) and QD (B), of average radii $\bar{a}_{1}=4.4 \mathrm{~nm}$ on the spacing $D$ between the QD $(A)$ and QD (B) surfaces. Here, $a_{e x}^{0}=0.38 \mathrm{~nm}(2)$ is the Bohr radius of a $2 \mathrm{D}$ exciton (formed by an electron and a hole spaced from the electron). 